\section{Suchttherapietage in Hamburg, 13.-16. Juni 2000}

\author{
Astrid Kühne \\ Zentrum für Interdisziplinäre Suchtforschung der Universität \\ Hamburg (ZIS)
}

Im Mittelpunkt des diesjährigen viertägigen Seminarkongresses Suchttherapietage in Hamburg stand die Auseinandersetzung mit dem Thema: „Medikamente in der Suchttherapie Stellenwert und Perspektiven“. Welchen Stellenwert sollen Präparate wie Methadon, Polamidon, Codein, Buprenorphin oder Heroin haben? Und: Welche Wahl hat der Abhängige?

Mehr als 600 Praktiker und Wissenschaftler aus mehreren europäischen Ländern diskutierten u.a. die Grenzen medikamentöser Strategien in der Suchtbehandlung (Dr. Gerhard Bühringer), beschäftigten sich mit der Frage, ob medikamentöse Behandlung das Ende der psychosozialen Ära bedeutet (Professor Dr. Dr. Wolfgang Schneider), und stritten, ob es „Suchtmittel für Süchtige“ im Sinne einer Substitution mit Originalstoffen geben sollte (Professor Dr. Jobst Böning versus Professor Dr. Michael Krausz). Eine besondere Perspektive auf das Thema „Medikamente“ bot die Veranstaltung „Doping Drogen - Olympische Spiele“. Mit den ehemaligen Spitzensportlern Rolf Danneberg und Karen König, den Journalisten Peter Unfried (taz) und Jörg Winterfeldt (Die Welt) sowie dem Publizisten Günter Amendt wurden in einem lebendigen Forum Fragen nach dem Zusammenhang von Doping, Drogenkonsum und gesellschaftlicher Entwicklung diskutiert.

Gelegenheit zum weiteren Erfahrungsaustausch bot das vielfältige Spektrum von über 100 Seminaren und Workshops unterschiedlicher suchttherapeutischer Behandlungsformen, beispielsweise Familienarbeit, Sucht und Angst, Psychoedukation bei Alkohol- und Drogenabhängigen, Kinder in Suchtfamilien, Suchtprävention an Schulen, Sucht im Alter und tagesklinische Behandlungen bei Alkohol-, Medikamenten- und Drogenabhängigkeit.

Die 6. Suchttherapietage vom 5.-8. Juni 2001 in Hamburg werden sich im Schwerpunkt mit dem Thema „Suchthilfe zwischen Psychotherapie und Safer Use“ befassen. Der vielfach chronische Verlauf der Suchterkrankung und die begleitenden körperlichen, psychischen und sozialen Probleme haben den Stellenwert des „klassischen“ Behandlungskonzepts Beratung - Entgiftung - (stationäre) Therapie relativiert. Zunehmend wichtiger werden suchtbegleitende, vorwiegend ambulante Konzepte - sei es als niederschwellige Kontaktund Zugangsinstitution, Angebote zur Krisenintervention, in Ergänzung medikamentöser Behandlungen oder als (eigenständige) psychosoziale Therapieform.

Darüber hinaus werden erneut aus dem gesamten Spektrum der Suchtarbeit verschiedene grundlegende sowie aktuelle Therapieformen zur Diskussion gestellt.
Bis zum 1.11.2000 können Beiträge zum Schwerpunkt oder zu Therapieformen angemeldet werden. Wenn Sie Interesse haben, einen eigenen Beitrag anzumelden, wenden Sie sich bitte an das:

\section{Kongressbüro Suchttherapietage 2001}

Zentrum für Interdisziplinäre Suchtforschung der Universität Hamburg (ZIS)

Zentrum für Psychosoziale Medizin, Psychiatrie und

Psychotherapie am UKE

Martinistraße 52

20246 Hamburg

Tel. 040/46070374

Fax 040/428035121

\section{KFH NW bildet Suchttherapeuten aus}

Die Katholische Fachhochschule Nordrhein-Westfalen (KFH NW) bildet erstmals Suchttherapeuten aus. Der dreijährige berufsbegleitende Kurs „Suchttherapeut/in KFH NW“ bildet nach den Richtlinien des Verbandes Deutscher Rentenversicherungsträger (VDR) Sozialarbeiter, Sozialpädagogen, Psychologen und Ärzte in Suchttherapie weiter. Kooperationspartner der KFH NW bei diesem Projekt sind der Sozialdienst Katholischer Männer (SKM) Köln und der Diözesan-Caritasverband Köln. Die Weiterbildung ist verhaltenstherapeutisch ausgerichtet mit kognitiven und systemischen Schwerpunkten und beginnt im Oktober in Köln.

Informationen und Anmeldung bei:

Prof. Dr. Michael Klein

KFH NW Abteilung Köln

Wörthstraße 10

50668 Köln

E-mail: Mikle@kfhnw-koeln.de

Tel. „Weiterbildungs-Hotline“ montags von 9 bis $10 \mathrm{Uhr}$ unter 0221/7757-155

Informationen im Internet unter: www.kfhnw.de 


\section{Mitteilungen der Deutschen Gesellschaft für Suchtpsychologie (DG SPS)}

\author{
Michael Klein \\ Katholische Fachhochschule Nordrhein Westfalen, \\ Fachbereich Sozialwesen, Köln
}

Im Berichtszeitraum seit April 2000 hat die Deutsche Gesellschaft für Suchtpsychologie (DG SPS) eine erfreuliche Entwicklung genommen. Das Interesse bei den Fachkolleginnen und Fachkollegen ist größer als erwartet. Wenige Monate nach ihrer Gründung hat die Gesellschaft bereits mehr als 80 Mitglieder, viele davon in leitenden Positionen der Suchthilfe oder seit langen Jahren in der Suchtforschung tätig. Der bis zum Oktober 2000 gewählte vorläufige Vorstand hat daher beschlossen, die Gesellschaft in Zukunft besonders für junge Berufskolleginnen und -kollegen attraktiv zu machen. Hierfür soll u.a. ein Curriculum zur Erlangung des Fachkundenachweises in Suchtpsychologie/Suchtpsychotherapie erarbeitet und etabliert werden.

Außerdem wurde die Bildung eines Arbeitskreises „Leitende Psychologen“ innerhalb der DG SPS beschlossen. Dieser soll sich mit den speziellen Anliegen und Fragestellungen der Fachkolleginnen und -kollegen in Leitenden Positionen in der Suchthilfe und -therapie beschäftigen.

Für den Mai 2001 wird ein Symposion zur Kontroverse um das kontrollierte Trinken geplant. Näheres kann hierzu in der nächsten Ausgabe der Suchttherapie mitgeteilt werden.

Am Freitag, dem 6. Oktober 2000, findet von 11.00 bis 15.00 Uhr die nächste Mitgliederversammlung am Psychologischen Institut der Universität Heidelberg (Lehrstuhl: Prof. Dr. J. Funke) statt. Die MV tagt im Übungsraum B in der Hauptstraße 47-51. Bei dieser soll der Vorstand neu und auf Dauer gewählt werden. Außerdem werden die Themen „Fachkundenachweis Suchtpsychologie“ und die Jahresplanung 2001 behandelt. Neben den Mitgliedern sind interessierte Fachkolleginnen und -kollegen herzlich eingeladen. Wie bereits üblich, trifft sich das „Netzwerk Psychologische Suchtforschung“ im Vorfeld, nämlich am Vorabend ab 17.00 Uhr, ebenfalls im Übungsraum B des Psychologischen Instituts der Universität Heidelberg.

Inzwischen ist die Web-Seite der DG SPS installiert und erfreut sich regen Zuspruchs. Sie ist unter: www.suchtpsychologie.de erreichbar. Informationen und Anregungen hierzu können an den Webmaster (Adresse ebendort verzeichnet) gesandt werden.

\section{Prof. Dr. Michael Klein}

Katholische Fachhochschule Nordrhein-Westfalen

Fachbereich Sozialwesen

Abteilung Köln

Wörthstraße 10

50668 Köln

Tel. (0221) 7757156

Fax (0221) 7757180

\section{BUCHBESPRECHUNGEN}

Uchtenhagen, Ambros und Zieglgänsberger, Walter (Hrsg.): Suchtmedizin - Konzepte, Strategien und therapeutisches Management. 2000; 626 S., DM 248,(Urban und Fischer, München, Wien) ISBN 3-437-21780-1 Ein wahrlich schwergewichtiges Vielmänner- und frauenwerk, das die beiden Herausgeber nach mehr als dreijähriger Arbeit vorlegen. Anders als der Titel „Suchtmedizin“ vermuten lässt, bemühten sie sich, die gesamte Palette interdisziplinärer Fragestellungen aus Epidemiologie, Pharmakologie, Psychologie, Sozialwissenschaften und Suchttherapie abzuarbeiten, ähnlich wie schon schwergewichtige Vorgänger aus anderen Verlagen. Es gehört aber wahrscheinlich auch zu den schwierigsten Aufgaben, für derartige Überblicks- und Handbücher Titel zu finden, die den Spagat bewältigen zwischen ausreichender Spezifität interessant zu bleiben und gleichzeitig eine ausreichende Plattform zu bieten. Die Herausgeber stehen zur Qualität in ganz unterschiedlichen Bereichen. Ambros Uchtenhagen als Grand Seigneur der europäischen Suchtforschung, als Direktor des Zürcher Instituts für Suchtforschung ist insbesondere mit versorgungsorientierten Fragestellungen befasst und einer besonderen Expertise im Bereich der Substitutionstherapie und der Heroinforschung. Zieglgänsberger wirkt als Pharmakologie- und ausgewiesener Grundlagenforscher am Max-Planck-Institut in München. Die restliche Autorenschar vereinigt so ziemlich alles, was im deutschsprachigen Raum im Zusammenhang mit dem Thema Sucht Rang und Namen hat. Es ist ein grundsätzliches Problem in Handbüchern, dass nicht alle Kapitel qualitätsmäßig zu den hohen Erwartungen passen. So reicht auch in dem vorliegenden Werk die Qualität von exzellenten Handbuchkapiteln in nahezu vollständiger Übersicht über die aktuelle Literatur und vollständiger konzeptioneller Darstellung (z.B. das Kapitel von Legnaro zur Kulturgeschichte oder das Kapitel der Psychopharmakologie und dem Reward System von Zieglgänsberger), über stichwortartige Übersichten, die die selektive Neugierde der geschätzten Leser nicht zu befriedigen vermögen, bis zu zwar volumigen, aber wenig substantiellen Darstellungen, bei denen man sich als respektloser Rezensent fragt, wann sich der Betreffende das letzte Mal mit der aktuellen Literatur und dem neuesten Forschungsstand in diesem Themenbereich beschäftigt hat (z.B. Petzold, Psychotherapie). Die durchschnittliche Qualität der Kapitel und deren lektorale Aufbereitung und Präsentation ist allerdings ansprechend und das, was man aufgrund der Herausgeber und Autoren erwarten würde. Wer sollte sich also für das Werk interessieren und die doch nicht unbeträchtliche Investition tätigen? Auf jeden Fall nicht nur Suchtmedizinerinnen und -mediziner, wenn diese aufgrund des Zuschnittes sowie der Preisgestaltung des Buches sicher eine der Hauptinteressentengruppen sein könnten. Es ist auch als Orientierungshilfe im Rahmen von therapeutischen Teams und Einstieg in die Vorbereitung für Dissertationen u.a. in diesem Themengebiet genauso interessant wie für Kolleginnen und Kollegen aus der Psychologie und Sozialarbeit. Ich fände es schön, wenn solche Bücher als preiswertere Paperbacks auch weniger gut Verdienenden zur Verfügung stehen würden wie z.B. das Kompendium für Psychiatrie und Psychotherapie im Karger-Verlag, das mit einem ähnlichen Volumen aufzuwarten hat (Thomas Spoerri: Kompendium der Psychiatrie und Psychotherapie, Hrsg.: Freyberger HJ und Stieglitz RD, 1996, Karger).

M. Krausz, Hamburg

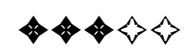

